\title{
Congenital Goiter Found in a District of Omuro, Kochi, Shikoku, Japan: Morphological Study
}

\author{
UICHIRO TEZUKA, TSUYOSHI MURAKAMI, KUNIO MISHIRO, \\ MASAAKI FUJINO AND OSAMU TAKEICHI \\ Department of Internal Medicine, School of Medicine, \\ Tokushima University, Tokushima
}

\begin{abstract}
Synopsis
Omuro goiter is a congenital condition characterized by euthyroidism with a high familial incidence which was discovered in a fishing village in Kochi Prefecture, Japan. It was reported previously that the goiter may have been caused by a disturbance in the biosynthesis of thyroid hormone which was characterized by reduced formation of $T_{4}$ and $T_{3}$. This might suggest the presence of a defect of coupling of iodotyrosines to form iodothyronines. This paper reports morphological studies on 11 cases of Omuro goiter.

Characteristic features of the goiter seen by light microscopy were parenchymatous and adenomatous hyperplasia of the thyroid tissue associated with microfollicles containing a little colloid. Follicular cells were mostly cylindrical in shape.

Electron microscopy showed that the follicular lumen contained colloid of low density. Fine microvilli were found on the free luminal surface of the cylindrical follicular cells. The rough-surfaced endoplasmic reticulum was greatly dilated expanding into almost the entire cytoplasm. Mitochondria were scattered among the endoplasmic reticulum and some cells contained many fat droplets. Lysosomes varying in size and electron density were seen in the supranuclear region. The follicular cells contain a few secretory granules of low density but no colloid droplets. The Golgi apparatus was atrophic and inconspicuous. Transport of newly synthesized thyroglobulin from the rough endoplasmic reticulum into the Golgi apparatus may be partly inhibited by the weak development of Golgi apparatus, and this fact may bring about the extraordinary dilatation of cisternae of the rough-surfaced endoplasmic reticulum. Well developed endoplasmic reticulum indicates the active synthesis of thyroglobulin probably accelerated by the stimulation of TSH, whose secretion is increased.
\end{abstract}

Omuro goiter was discovered in the Omuro Community, a small fishing village in Kochi Prefecture. It is a simple goiter characterized by euthyroidism and has a high congenital and familial incidence. A series of studies on iodine metabolism in this goiter disclosed that there was no disturbance in iodide accumulation, iodide organization, the iodotyrosine dehalogenase reaction or formation of a plasma iodoprotein. It was presumed that there might be presence of a defect of

Received for publication June 1, 1971. coupling of iodotyrosines to form iodothyronines. In previous papers the authors (Tezuka et al., 1970 and 1971) reported that goiters show dominant autosomal heredity of incomplete penetrance in view of their high familial incidence, but there are no reports on the occurrence of this type of goiter, supposedly due to a congenital disorder of iodine metabolism.

This paper on the morphology of Omuro goiter describes the pathological changes in ultrastructure of the goiter observed by electron microscopy as well as the result of a gross light microscopic observation. 


\section{Materials and Methods}

A small portion of Omuro goiter tissue was removed from each of 11 cases ( 5 males and 6 females; 8 cases of diffuse type and 3 cases of nodular type) of the 16 cases who entered Tokushima University Hospital for examination. Specimens from 10 cases ( 5 males and 5 females; 7 cases of diffuse type and 3 cases of nodular type) were examined by electron microscopy.

Specimens for examination by light microscopy were fixed in $10 \%$ formalin solution immediately after removal, embedded in paraffin wax cut into sections and stained with hematoxylin-eosin or periodic acid Shiff reaction (PAS).

Specimens for the electron microscopic study were cut into pieces less than $1 \mathrm{~mm}^{3}$ in size and fixed at $4^{\circ} \mathrm{C}$ for $2 \mathrm{hr}$ in $1 \%$ osmium tetroxide solution buffered at pH 7.4 with veronal acetate. Then sections were rinsed gently with distilled water, dehydrated with an aceton series (Palade's method) (1952), and embedded in epoxy resin following Kushida's method (1960). Ultra-thin sections were cut with a Porter-Blum microtome and stained with lead acetate (Millonig's method) (1961). They were examined with Hitachi HU-11S and HS-7 type electron microscopes. Micrographs were taken at a direct magnification of 3,000 5,000 times.

\section{Results}

\section{Light microscopic findings}

Under the light microscope, Omuro goiter tissue appeared to be characterized by parenchymatous hyperplasia associated with dominant microfollicles as shown in Figure 1, while macrofollicles are also seen as shown in Figure 2. Both the macro-, and microfollicles contained a little colloid, which was weakly positive in PAS reaction. These follicles were lined almost entirely with cylindrical epithelial cells with a few cuboidal or flat cells. Some cells stained poorly due to a remarkable vacuolar degeneration.

There was no evidence of round cell infil-

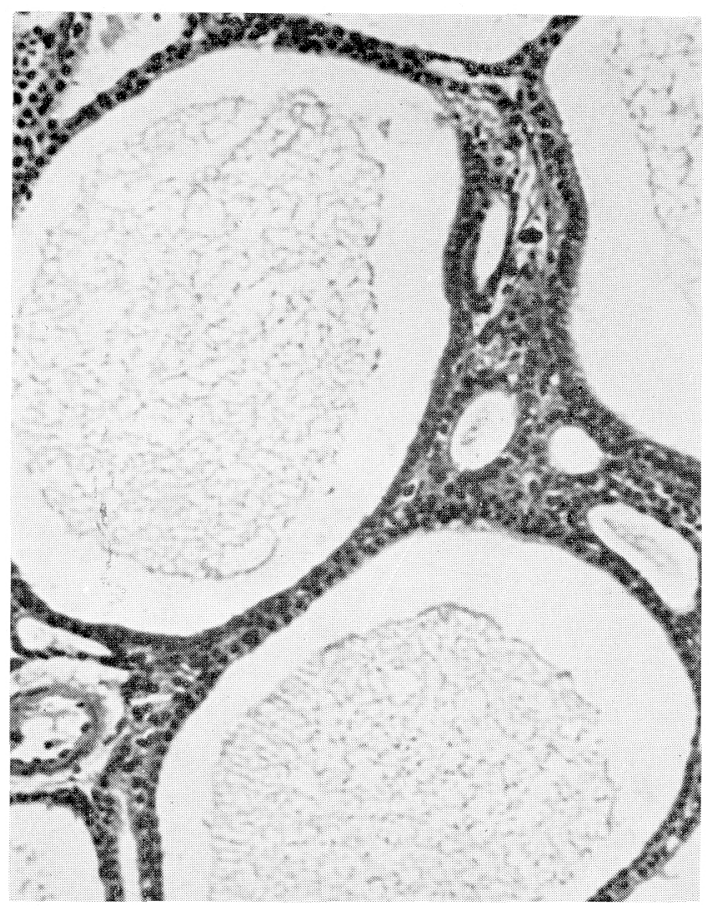

Fig. 2. Light micrograph of Omuro goiter tissue with macrofollicles. Stain; Hematoxylin-eosin. $\times 100$ 


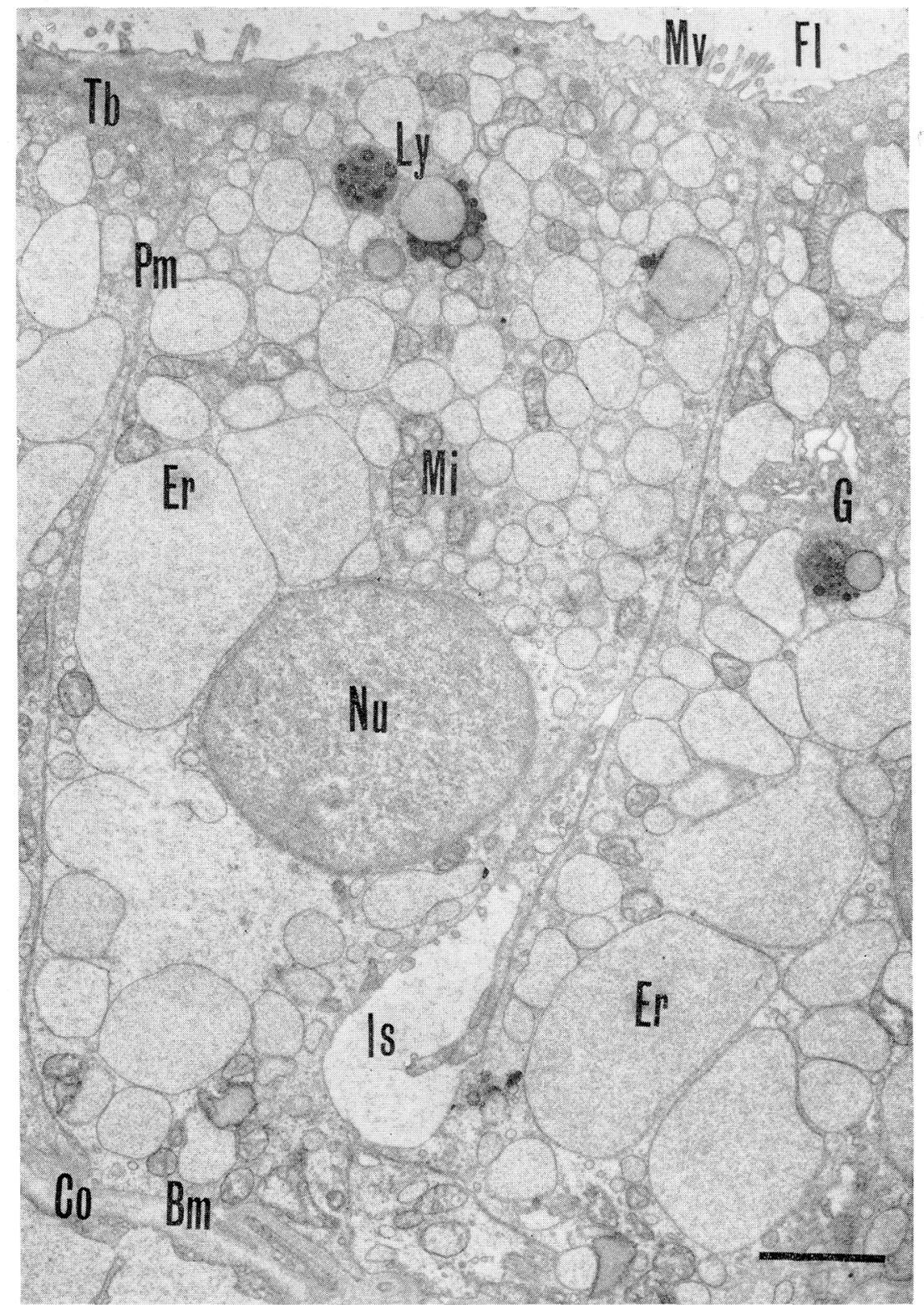

Fig. 3. Electron micrograph of cylindrical follicular cells of Omuro goiter. $\quad \times 11,000$ Fl: follicle lumen, Mv: microvilli, Tb: terminal bar, Pm: plasma membrane, Mi: mitochondria, G: Golgi apparatus, Er: rough-surfaced endoplasmic reticulum, Nu: nucleus, Is: intercellular space, $\mathrm{Bm}$ : basement membrane, Co: connective tissue, Ly: lysosome.

tration or appreciable fibrosis of the connective tissue stroma.

No malignancy was found in any case.

From the histological studies, 9 cases of
Omuro goiter were diagnosed as microfollicular goiter, 1 as micro-macrofollicular goiter and 1 as macrofollicular goiter. The histological findings show that this disease can 


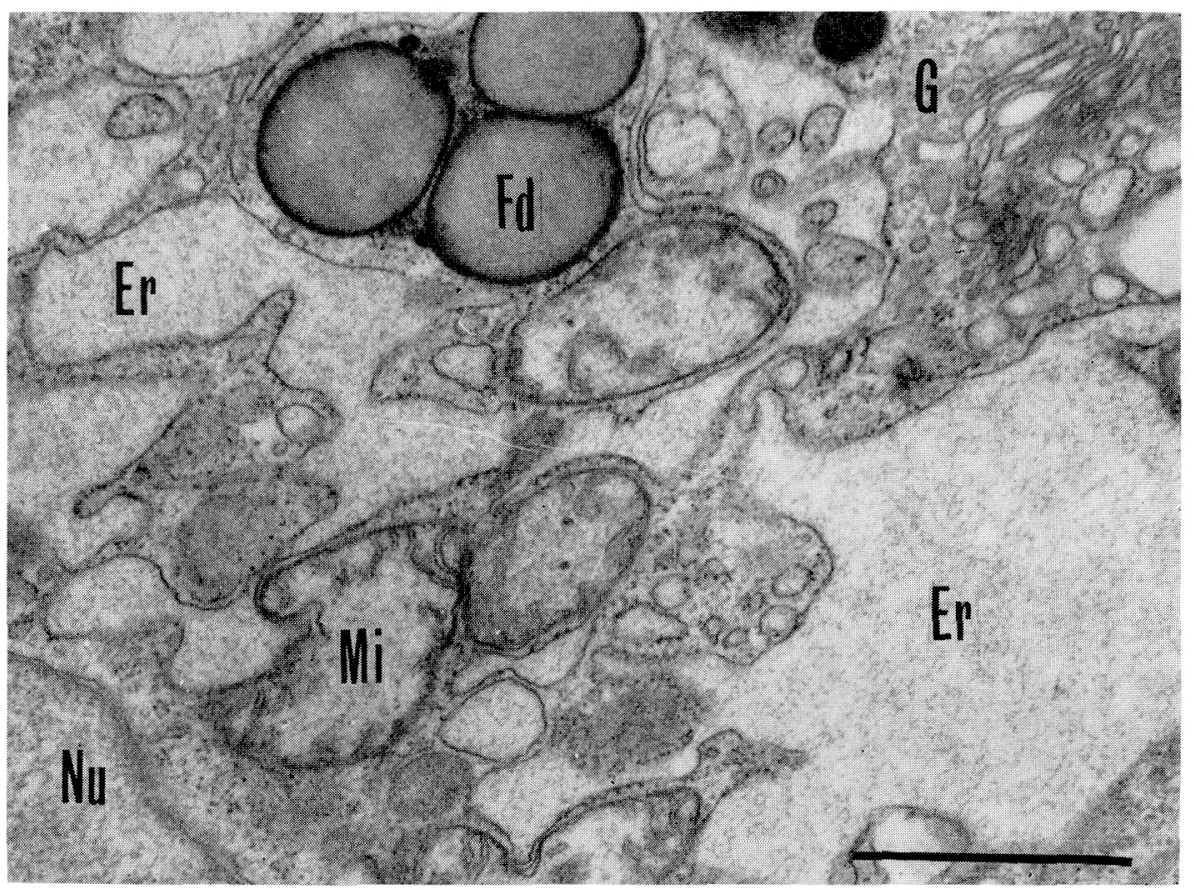

Fig. 4. Dilated cisternae of the rough-surfaced endoplasmic reticulum in a follicular cell. $\times 28,000$

Fd: fat droplet. Other symbols are the same as in Fig, 3.

be classified as a type of adenomatous hyperplasia.

\section{Electron microscopic findings}

Electron microscopy of ultrastructure of Omuro goiter demonstrated that follicles in most cases were surrounded by cylindrical follicular cells and contain colloid of less electron density in the follicular lumen. (Fig. 3)

There were fine microvilli on the free luminal surface of the follicular cells.

The rough-surfaced endoplasmic reticulum was greatly expanded especially in the basal zone and occupied almost the entire cytoplasm. The dilated cisternae of the rough endoplasmic reticulum contained a substance of low electron density like that found in the follicular lumen. In some cells cisternae of the endoplasmic reticulum were so much dilated that they adhered and fused to each other to form larger cisternae. (Fig. 4) Mithochondria were scattered among the endoplasmic reticulum and there were many fat droplets in some cells. (Fig. 5) In the supranuclear region, primary and secondary lysosomes with vacuoles and granules were seen. These lysosomes differed from one another in electron density and in size. There were also a few fine secretory granules of low electron density, but no colloid droplets were seen as large less dense granules. (Fig. 6)

The Golgi apparatus was generally atrophic and inconspicuous. (Fig. 7)

The nuclei were round or oval in form and bounded by the double nuclear membranes. The karyoplasm seemed to consist of fine particles and contained the nucleolus of rather high electron density.

The desmosomes were also recognized in some places along the cell boundary between the adjacent follicular cells. Well developed 


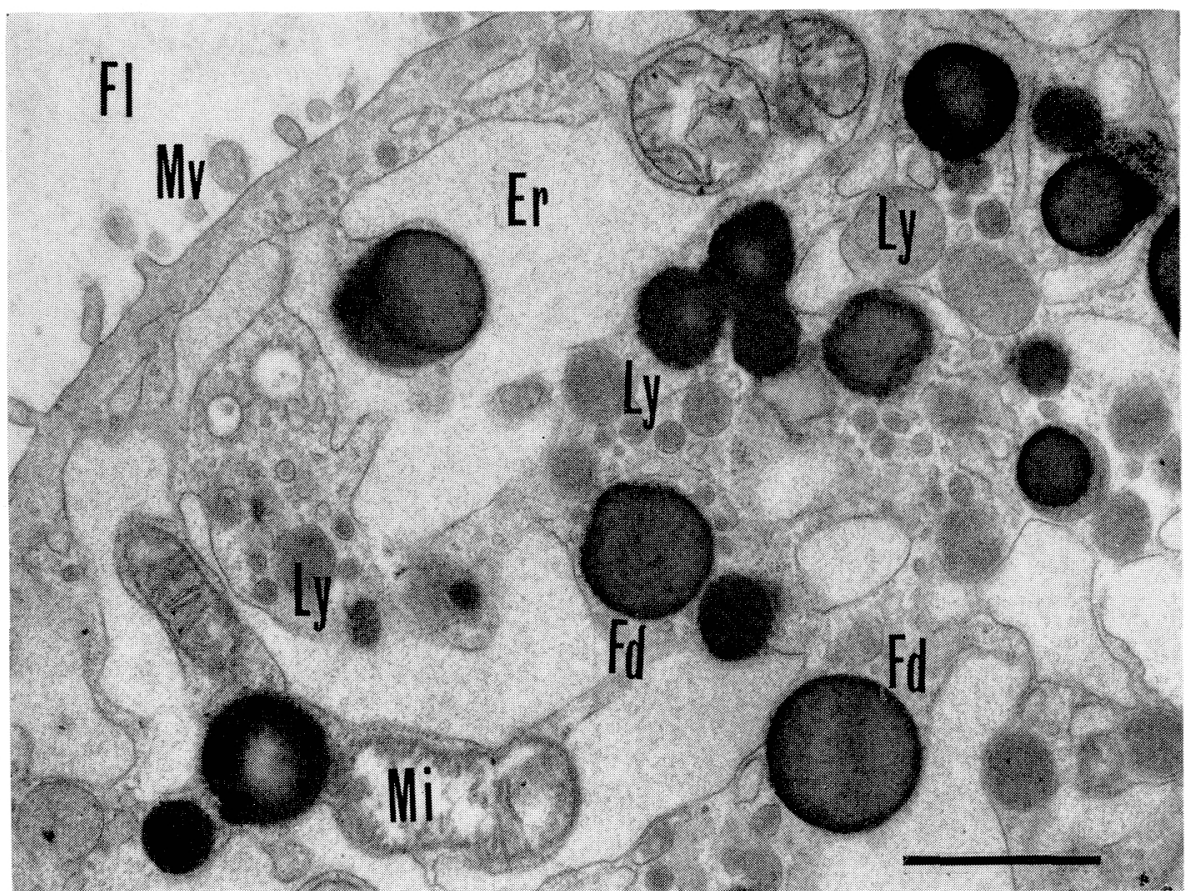

Fig. 5. Dilated cisternae of the rough-surfaced endoplasmic reticulum, many lysosomes and fat droplets in a follicular cell. $\times 18,000$

Symbols are the same as in the previous figures.

terminal bars were found at the uppermost part of the lateral cell surface. Occasionally intercellular spaces were found in the basal zone of the follicular epithelium and contained a less dense material than that of the follicular lumen. The basement membrane was present outside the plasma membrane of the basal cell surface which showed a markedly tortuous coarse. There were connective tissue and capillary endothelial cells beneath the follicular epithelium.

Some follicular cells were cuboidal or flat in appearance. In these cases, colloid in the follicular lumen also had low electron density, though microvilli of the follicular cells were slightly thicker and fewer in number than those of cylindrical cells. Cisternae of the rough-surfaced endoplasmic reticulum were greatly dilated, and contained a material of low electron density. There were many lysosomes in the supranuclear region, but few secretory granules and no colloid droplets. The Golgi apparatus was atrophic and inconspicuous.

\section{Discussion}

In case reports of sporadic cretinism due to coupling defect, Stanbury et al. (1963), Werner et al. (1957) and Mosier et al. (1958) described the histological features of the goiter in this condition. According to their reports, the histology of the goiter in most cases showed adenomatous and papillary hyperplasia, but some cases were complicated with fetal, trabecular or tubular adenoma. The histological features of all the present 11 cases of goiter were also those of adenomatous hyperplasia and most of them had microfollicles. However, examination was so limited to the small portions of the thyroid obtained by 


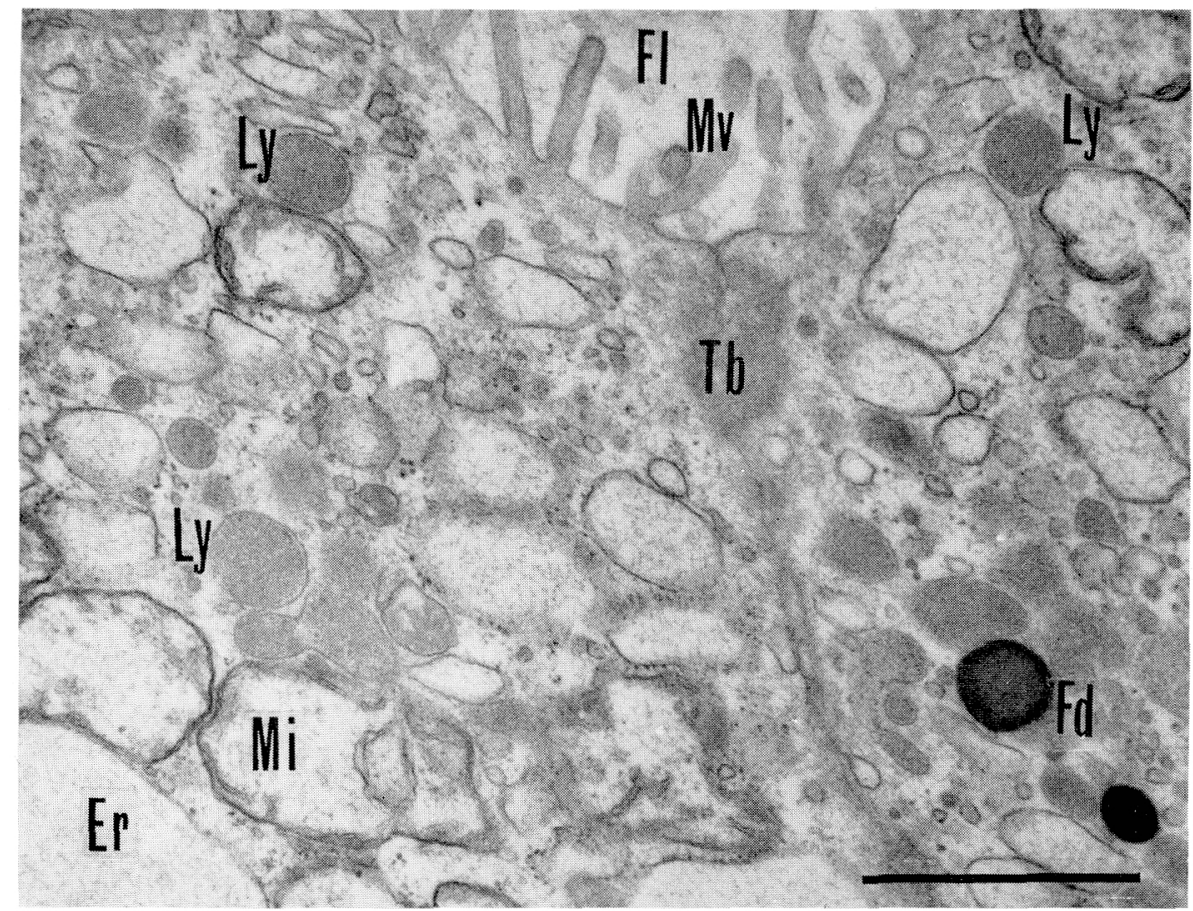

Fig. 6. Supranuclear region of a follicular cell. $\times 28,000$ Symbols are the same as in the previous figures.

incisional biopsy, that it is uncertain whether the goiter was complicated by adenoma, carcinoma or chronic inflammation.

The results of electron microscopy on the human thyroid gland have been reported by Walthard (1955), Garnier (1956), Irie (1960), Niwa (1963), Heimann (1966) and Lupulescu and Petrovici (1968). Furthermore, there have been no previous reports of electron microscopic studies of goiters associated with impaired biosynthesis of thyroid hormone due to a coupling defect.

The most striking electron microscopic finding in this goiter is the remarkable expansion of cisternae of the rough-surfaced endoplasmic reticulum containing a material of low electron density in the cytoplasm of cylindrical follicular cells.

According to Kurosumi (1961 and 1966), secretory protein may be synthesized in the cytoplasm on the ribosomes located outside the endoplasmic reticulum by the instruction of messenger RNA derived from the nucleus. Synthesized protein is said to be accumulated in the cisternae of the endoplasmic reticulum which are separated from the ribosomes by a membrane. Dempsey and Peterson (1955) Wang (1958) and Fujita et al. (1958) also stated that thyroglobulin is synthesized in the rough-surfaced endoplasmic reticulum. Therefore, expansion of cisternae of the endoplasmic reticulum may indicate that protein synthesis is greatly increased by some stimuli. Expanded cisternae have been found by electron microscopy of the thyroid gland of patients with Graves disease (Garnier, 1956; Irie, 1960; Niwa, 1963; Heimann, 1966; Luplescu and Petrovici, 1968) and in the thyroid of animals after administration of TSH (Braunsteiner et al., 1953; Yoshimura and Irie, 1959), thiouracil (Braunsteiner et al., 1953; Dempsey and Peterson, 1955; Yoshimura and Irie, 


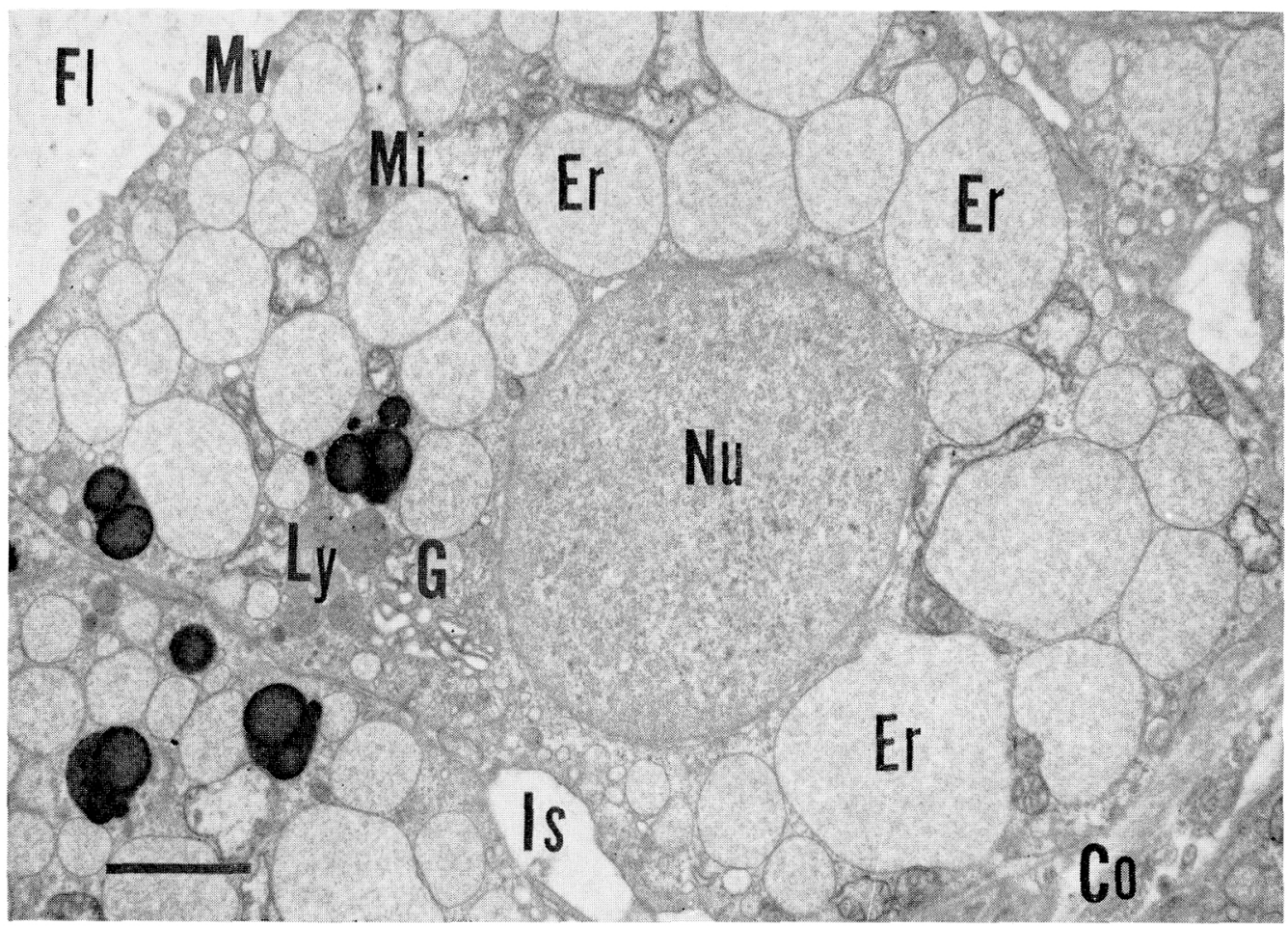

Fig. 7. Atrophic Golgi apparatus in a follicular cell. $\times 15,000$ Symbols are the same as in the previous figures.

1959) or adrenalin (Fujita et al., 1958). The expansion of cisternae of the endoplasmic reticulum found in the follicular cells of Omuro goiter seems to be brought about by a strong stimulus probably due to an increased secretion of endogenous TSH.

Protein synthesis in the endoplasmic reticulum seems to be increased, but relativey low electron density of the colloid in the follicular lumen indicates a possibility that the concentration of colloid might be low. These features suggest the following two possibilities. 1) The thyroglobulin synthesized in the endoplasmic reticulum might be incomplete in quality; 2) there might be more or less inhibition in transport of thyroglobulin during the process of secretion into the follicles.

The first possibility was disproved as described in detail in the previous report (Tezuka et al., 1971).

According to Kurosumi (1961 and 1966), hormonal protein accumulating in the endoplasmic reticulum is transported into the Golgi apparatus and forms secretory granules of uniform size and electron density. It is reported that mature secretory granules may leave the Golgi apparatus, migrate toward the periphery of the cell, become attached to the surface of the plasma membrane and be released from the cells mainly through a mechanism of the so-called reverse pinocytosis (eruptocrine secretion).

In Omuro goiter, however, the Golgi apparatus was poorly developed and indistinct, while the endoplasmic reticulum was greatly expanded. Moreover, there were few secretory granules formed in the Golgi apparatus. Thus it seems that there is a little release of secretory granules into the follicular lumen, resulting in the poor accumulation of colloid. Colloid droplets which are considered to be an indication of reabsorption of 
colloid from the follicles (Sheldon et al., 1964; Seljelid, 1967) and which are frequently seen in cases of Graves desease (Garnier, 1956; Irie, 1960; Niwa, 1963; Heimann, 1966; Luplescu and Petrovici, 1968), were not observed in the present cases.

Therefore, the decrease of colloid secretion despite the expansion of the endoplasmic reticulum suggesting an increased thyroglobulin synthesis seems to be brought about by a relative inhibition of transportation from the endoplasmic reticulum into the Golgi apparatus.

Wollman et al. (1964) reported that, when the gland was stimulated by TSH, many lysosomes containing hydrolytic enzyme appeared in the supranuclear region and these were responsible for the hydrolysis of colloid in the liberation of thyroxin. In the cells of the pituitary of a thyroidectomized rat, Kurosumi (1968) considered that lysosome substance accumulated in the Golgi apparatus may impair the transport of secretory substance from the endoplasmic reticulum to the Golgi apparatus, resulting in a strong expansion of the endoplasmic reticulum.

From the present findings and discussions, it is concluded that there is a certain relative inhibition in the transport process of the secretion through the Golgi apparatus. This interfered secretory process of thyroid hormone may bring about the accelerated secretion of pituitary TSH, which in turn increased the protein synthesis in the thyroid contributing to the development of goiter.

\section{Acknowledgements}

The authors are very grateful to Prof. T. Aburaya of Tokushima University School of Medicine for his kind guidance in this investigation, and Prof. $K$. Kurosumi of Institute of Endocrinology, Gunma University, Maebashi, for suggestion in electron microscopic studies and critical reading of manuscript. They also thank Prof. K. Hizawa and Prof. H. Otsuka of Tokushima University School of Medicine for their advice in pathological work. They also wish to express their gratitude to Dr. Y. Sakakibara of Tokushima
University Hospital for his kind cooperation in surgery.

\section{References}

Braunsteiner, H., K. Fellinger and F. Pakesch (1953). Endocrinology 53, 123.

Dempsey, E. W. and R. R. Peterson (1955). Ibid. 56, 46.

Fujita, H., M. Kano and S. Okamoto (1958). Arch. Histol. Japon. 14, 61 (in Japanese).

Garnier, B. (1956). Schweiz. Z. Path. Bakt. 19, 129.

Heimann, P. (1966). Acta Endocrin. (Kobenhavn) 53, suppl. 110.

Irie, M. (1960). Arch. Hist. Japon. 19, 39 (in Japanese).

Kushida, H. (1960). J. Electron Micr. (Tokyo) 9, 157 (in Japanese).

Kurosumi, K. (1961). Int. Rev. Cytol. 11, 1.

Kurosumi, K. (1966). Protein, nucleic acid and enzyme (Tokyo) 11, 1278 (in Japanese).

Kurosumi, K. (1968). Clin. All Round (Osaka) 17, 1836 (in Japanese).

Luplescu, A. and A. Petrovici. Ultrastructure of the thyroid gland. S. Karger, Basel Ltd., New York, P. 64 (1968).

Millonig, G. (1961). J. Biophys. Biochem. Cytol. 11, 736.

Mosier, H. D., R. M. Blizzard and L. Wilkins, (1958). Pediatrics 21, 248.

Niwa, Y. (1963). Shinshu Med. J. 12, 207 (in Japanese).

Palade, G. E. (1952). J. Exp. Med. 95, 285.

Stanbury, J. B., G. Riccabona and M. A. Janssen (1963). Lancet 1, 917.

Sheldon, H., J. M. Mckenzie and D. van Nimwegan (1964). J. Cell. Biol. 23, 200.

Seljelid, R. (1967). J. Ultrastruct. Res. 17, 401.

Texuka, U., T. Murakami, M. Niki, K. Mishiro, M. Fujino and O. Takeichi (1970). Endocrinol. Japon. 17, 289.

Tezuka, U., T. Murakami, K. Mishiro, M. Fujino and O. Takeichi (1971). Ibid. 18, 265.

Werner, S. C., R. J. Block, R. H. Mandel and 
A. A. H. Kassenaar, J. Clin. Endocrinol. 17, 817.

Walthard, B. (1955). Bull. Schweiz. Akad. Med. Wiss. 11, 346.

Wang. L. (1958). Electron-Microscopy (To- kyo) 6, 184 (in Japanses).

Wollman, S. H., S. S. Spicer and M. S. Burstone (1964). J. Cell Biol. 21, 191.

Yoshimura, F. and M. Irie (1959). Clin. Endocrin. (Tokyo) 7, 367 (in Japanese). 